\title{
Finite Blocklength Analysis of the Multiple Access Relay Channel with Batch-And-Forward Strategy
}

\author{
Christos K. Kourtellaris, Constantinos Psomas, Ioannis Krikidis \\ Department of Electrical and Computer Engineering, University of Cyprus, Cyprus \\ Email: \{kourtellaris.christos, psomas, krikidis\}@ucy.ac.cy
}

\begin{abstract}
This work provides a comprehensive framework regarding the analysis of the Multiple Access Relay Channel (MARC) with Time Division Multiple Access (TDMA) scheduling, subject to finite blocklength constraints. In particular, we examine the stability conditions and evaluate the maximum throughput, by applying a cognitive cooperation protocol that assumes relaying is enabled when sources are idle. Moreover, we propose the novel Batch-And-Forward (BAF) strategy, that can significantly enhance the performance of cooperative networks in the finite blocklength regime, as well as reduce the requirement in metadata. The BAF strategy is quite versatile, thus, it can be embedded in existing cooperative protocols, without imposing additional complexity on the overall scheme.
\end{abstract}

Index Terms-Network stability, finite blocklength, cognitive cooperation, multiple access relay channels, finite blocklength analysis.

\section{INTRODUCTION}

Although Shannon's information theory has evolved over the years to include applications in a wide range of fields related to communications, it has failed to leave its distinct mark in the field of communication networks [1]. One of the main reasons that led to this result, is the asymptotic nature of information theory which cannot sufficiently address the finite blocklength constraints in communication applications [1], [2]. Fortunately, recent results [3]-[5] provide valuable tools regarding the analysis of communication networks in the finite blocklength regime. These results were applied to address the requirement of low latency from various perspectives, such as, the characterization of finite blocklength rates for various channels [6], [7], the performance evaluation of short length codes [8], and the performance analysis of communication protocols [9]. On topics related to cooperation in multiple access channels, though there is an extensive literature that spans from the performance analysis [10] to protocol design [11], [12], the vast majority of this literature regards asymptotic, in terms of blocklength, analysis. Thus, though the majority of these techniques can be employed in the context of finite blocklength, they do not necessarily perform in an optimal manner.

This work was supported in parts by the European Regional Development Fund and the Republic of Cyprus through the Research Promotion Foundation (Project: INFRASTRUCTURES/1216/0017) and in parts by the European Regional Development Fund and the Republic of Cyprus through the Research Promotion Foundation(Project: EXCELLENCE/1216/0365).
In view of the above limitations, we apply tools from information theory, stochastic processes and queueing theory, in order to provide a comprehensive framework regarding the performance analysis of these networks, in the finite blocklength regime. We apply the emerged framework in order to reexamine the stability of the Multiple Access Relay Channel (MARC) with Time Division Multiple Access (TDMA) scheduling. The selected cognitive cooperation protocol [10], which is based on the underlay cognitive radio concept, assumes relaying is enabled when sources are silent (idle). Although the cognitive cooperation protocol improves the performance of the network, this improvement is disproportionate to the additional complexity and resources that it entails. The reason for the insufficient performance is that existing cooperative techniques are not designed to perform optimally in the finite blocklength regime. Towards this direction, we propose a novel strategy that can significantly enhance the performance of networks that employ short codes.

The contributions of this paper are twofold. First, it characterizes the stability region and the maximum throughput of the MARC-TDMA network subject to finite blocklength constraints, for a particular cognitive cooperation protocol, and evaluates (numerically) the optimal trade-off between between data length and channel's blocklength. Second, it proposes the Batch-And-Forward (BAF) strategy which improves the performance of the network, in the finite blocklength regime. The main idea behind this strategy is the following. Keep the codeword blocklength fixed for all terminals in order to meet the practical requirement for fixed codeword blocklength, but allow each terminal to batch more than one data packet in each codeword. The BAF strategy is embedded in the discussed cognitive cooperation protocol, where we evaluate the stability conditions of the overall scheme and show, via numerical evaluation, that the overall performance is significantly enhanced. Although the performance is evaluated for a particular cooperative protocol, the proposed strategy is quite versatile, thus, it can be embedded in the majority of existing cooperative techniques, without imposing additional complexity.

The remainder of this paper is organized as follows. In Section II, we briefly review the recent results in the finite blocklength analysis, in Section III, we examine the cooperation in the finite blocklength regime, and in Section IV, we examine and discuss the proposed BAF strategy. 


\section{PRELIMINARIES ON FINITE BLOCKLENGTH ANALYSiS}

Despite the tremendous theoretical importance of Shannon's capacity, the prerequisite of infinite length codes severely limits its practical importance. This limitation becomes even more critical for communication applications where finite blocklength is imperative. The above problem can be addressed via the optimal fixed blocklength rate, $R^{*}(n, \varepsilon)$, where $n$ is the packet length (channel's blocklength) and $\varepsilon$ the packet error probability, which eliminates the necessity of infinitely large codes imposed directly by the definition of capacity. While, in general, $R^{*}(n, \varepsilon)$ is an NP-hard problem [2], [13], a recent work [3], among others, refines Strassen's normal approximation of $R^{*}(n, \varepsilon)$ [14], and provides an attractive tight approximation for it. In particular, they proved that for a class of channel models with positive capacity, $C, R^{*}(n, \varepsilon)$ is given by

$$
R^{*}(n, \varepsilon)=C-\sqrt{\frac{V}{n}} Q^{-1}(\varepsilon)+\mathcal{O}\left(\frac{\log n}{n}\right),
$$

where $C$ is the ergodic capacity, $V$ is the channel's dispersion [3], $Q^{-1}(\cdot)$ is the inverse of the Gaussian Q-function and $\mathcal{O}(\log n / n)$ comprises of the higher order terms. For a review of finite blocklength rate for various channels, see [2] and references within. In particular, for the Additive White Gaussian Noise (AWGN) channel, the channel's capacity and dispersion are given by

$C=\frac{1}{2} \log _{2}(1+S N R)$ and $V=\frac{S N R}{2} \frac{S N R+2}{(S N R+1)^{2}}\left(\log _{2} e\right)^{2}$,

respectively, where $S N R$ denotes the signal to noise ratio. The finite blocklength rate subject to equal-power constraint is then approximated by

$$
R^{*}(n, \varepsilon) \approx C-\sqrt{\frac{V}{n}} Q^{-1}(\varepsilon) .
$$

Substituting $R^{*}(n, \varepsilon)=\frac{k}{n}$, where $k$ denotes the size of the data packet, and solving with respect to the packet error probability $\varepsilon$, we obtain

$$
\varepsilon(k, n) \approx Q\left(\frac{n C-k}{\sqrt{n V}}\right) .
$$

The probability of successful transmission for a code of blocklength $n, P_{c}(k, n)$, is the cumulative distribution function (cdf) of the normal distribution, and it is expressed as

$$
P_{c}(k, n)=1-\varepsilon(k, n) \approx \frac{1}{\sqrt{2 \pi}} \int_{-\infty}^{\frac{n C-k}{\sqrt{n V}}} e^{-\frac{z^{2}}{2}} d z .
$$

The recent work in [4], refined the approximation given in (3), by providing the third order term in the normal approximation for the AWGN channel, that resulted in tighter expressions.

\section{COOPERATION IN THE FINITE BLOCKLENGTH REGIME}

In this section, we examine a packet-based network cooperation scenario with bursty arrivals at the source terminals. In particular, we consider a MARC scheduling and evaluate the

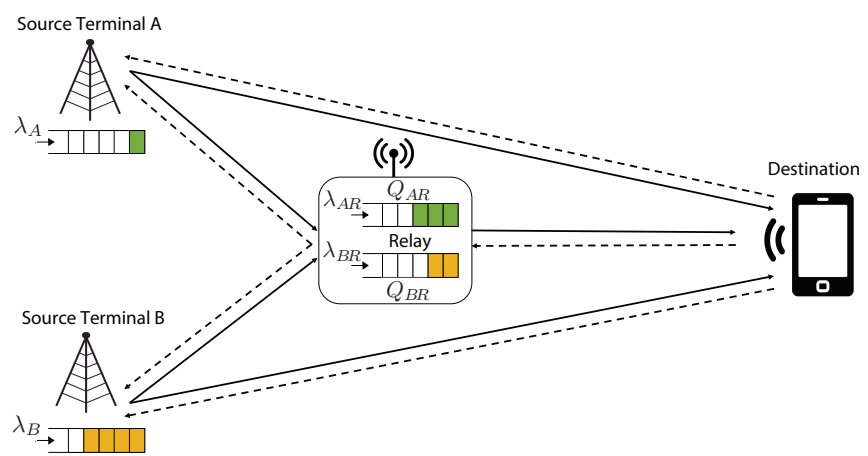

Fig. 1: Model of a MARC-TDMA network. The solid arrows represent the AWGN channels and the dashed arrows the ACK/NACK feedback.

performance of a cognitive cooperative protocol in the finite blocklength regime.

The MARC configuration is consisted of two source terminals, $A$ and $B$, a common cognitive relay and a destination, as depicted in Fig. 1. The data packets arrive at the source terminals, $A$ and $B$, according to independent and stationary Bernoulli processes with probabilities, $p_{A}$ and $p_{B}$, and expected values, $\lambda_{A}=p_{A}$ and $\lambda_{B}=p_{B}$, respectively. Each of the source terminals has an infinite size buffer memory, denoted by $Q_{i}, i \in\{A, B\}$, respectively, that stores the incoming data packets. The relay is equipped with two relaying queues, denoted by $Q_{A R}$ and $Q_{B R}$, in which they store the data packets received from the respective source terminals. Let $P_{c, S D}(k, n), P_{c, S R}(k, n)$ and $P_{c, R D}(k, n)$ denote the probability of a successful transmission from any source terminal $i \in\{A, B\}$ to the destination, from any source terminal $i \in\{A, B\}$ to the relay, and from the relay to the destination, respectively. The probabilities of erroneous transmissions are then defined by $P_{e, S D}(k, n)=1-P_{c, S D}(k, n)$ and $P_{e, S R}(k, n)=1-P_{c, S R}(k, n)$. Moreover, let $u^{C C}(k, n)$ and $u^{C C, *}(k, n)$ denote the throughput and the maximum throughput of the system, respectively, and $X^{C C}(k, n)$ denote the code rate of the overall scheme.

There is an extensive literature regarding multiple access protocols in the presence of a cooperating relays [10]-[12]. In this work we employ the cognitive cooperation protocol, defined below.

Definition 1. The Cognitive Cooperation (CC) protocol performs as follows:

i) Source terminal $i \in\{A, B\}$ encodes the data packet of length $k$ into a codeword of length $n$, and access the channel via a randomized TDMA scheduling with probability $\omega_{i}, i \in\{A, B\}$, and $\omega_{A}+\omega_{B}=1$.

ii) The codeword is transmitted both to the destination and the relay node. The transmission process is supported by an ACK/NACK mechanism that informs the source terminal and the relay about the transmission status (successful or erroneous). 
iii) If data are not successfully received by either the destination or the relay, the data packet remains in the queue of the source terminal.

iv) If data are successfully received by the destination, the source terminal removes the data packet from its queue, and the relay ignores the received packet.

v) If data are not successfully received by the destination but are successfully received by the relay, the source terminal discards the data packet from its queue, and the relay adds that packet to the respective queue $\left(Q_{A R}\right.$ or $\left.Q_{B R}\right)$.

vi) When the source terminal $i \in\{A, B\}$ gains access to the channel but it has no data packets in its queue (queue is idle), the relay encodes a data packet from the respective queue $Q_{i R}, i \in\{A, B\}$, into a codeword of length $n$, and transmits it to the destination.

This protocol, though not ideal in terms of performance, is attractive due to its elegance and simple structure, that allows the interpretation of the results in the context of finite blocklength codes. Nevertheless, the discussed methodology can be applied to more complex protocols.

\section{Theorem 1. For a fixed blocklength $n$ :}

i) the stability condition of the CC protocol is given by

$$
\begin{gathered}
\lambda_{A}+\lambda_{B}<u^{C C}(k, n), \\
\text { where } \\
u^{C C}(k, n) \triangleq \frac{k}{n} \frac{\left[P_{c, S D}(k, n)+P_{e, S D}(k, n) P_{c, S R}(k, n)\right] P_{c, R D}(k, n)}{\left[P_{c, R D}(k, n)+P_{e, S D}(k, n) P_{c, S R}(k, n)\right]} .
\end{gathered}
$$

ii) the code rate of the overall scheme, $X^{C C}(k, n) \triangleq \frac{k}{n}\left(\lambda_{A}+\right.$ $\left.\lambda_{B}\right)$, is bounded above by the maximum throughput $u^{C C, *}(k, n)$, that is

$$
X^{C C}(k, n)<\max _{k} u^{C C}(k, n) \triangleq u^{C C, *}(k, n) .
$$

Proof. i) The proof for the first statement consists of two parts: characterizing the stability regions for the source terminals and the relay, and evaluating the union of the predefined regions. Regarding the source terminals, the arrival rate at each source terminal is $\lambda_{i}, i \in\{A, B\}$, whereas the departure (service) rate is given by

$$
\mu_{i}=\omega_{i}\left[P_{c, S D}(k, n)+P_{e, S D}(k, n) P_{c, S R}(k, n)\right], \quad i \in\{A, B\} .
$$

The system at each source terminal $i$ forms a DTMC with stability condition $\frac{\lambda_{i}}{\mu_{i}}<1, \forall i \in\{A, B\}$ [15], or equivalently

$$
\lambda_{i}<\omega_{i}\left[P_{c, S D}(k, n)+P_{e, S D}(k, n) P_{c, S R}(k, n)\right], \forall i \in\{A, B\} .
$$

By recalling that $\omega_{A}+\omega_{B}=1$, the summation (10) over all source terminals $i \in\{A, B\}$ yields the following overall stability condition for the source terminals.

$$
\begin{aligned}
\Lambda_{S}^{C C}=\{ & \left(\lambda_{A}, \lambda_{B}\right):\left(\lambda_{A}+\lambda_{B}\right) \\
& \left.<\left[P_{c, S D}(k, n)+P_{e, S D}(k, n) P_{c, S R}(k, n)\right]\right\} .
\end{aligned}
$$

Regarding the relay, a packet from the source terminal $i, i \in$ $\{A, B\}$, enters queue $Q_{i R}$ at the relay if i) channel access for the source terminal $i$ is granted by the randomized switch (with probability $\omega_{i}$ ), ii) the transmission from the source terminal $i$ to the relay is successful, iii) the transmission from the source terminal $i$ to the destination is unsuccessful, and iv) the queue of the source terminal $i$ is not idle, that is, it has at least one packet that requires transmission. The source is not idle with stationary probability $1-\pi_{i, 0}$, where $\pi_{i, 0}=1-\frac{\lambda_{i}}{\mu_{i}}$ [15]. Thus, the rate of arrivals at relay's queue $Q_{i R}, i \in\{A, B\}$, is given by

$$
\lambda_{i R}=\omega_{i}\left(1-\pi_{i, 0}\right) P_{e, S D}(k, n) P_{c, S R}(k, n), \quad i \in\{A, B\} .
$$

Similarly, the average rate of departures from the relay's queue $Q_{i R}, i \in\{A, B\}$, is given by

$$
\mu_{i R}=\omega_{i} \pi_{i, 0} P_{c, R D}(k, n), \quad i \in\{A, B\} .
$$

The stability condition for the individual queue $Q_{i R}, i \in\{A, B\}$ at relay, is $\frac{\lambda_{i R}}{\mu_{i R}}<1, \forall i \in\{A, B\}$. By employing (9), (12) and (13), the stability condition, $\forall i \in\{A, B\}$, translates to

$$
\lambda_{i R}<\frac{\omega_{i}\left[P_{c, S D}(k, n)+P_{e, S D}(k, n) P_{c, S R}(k, n)\right] P_{c, R D}(k, n)}{\left[P_{c, R D}(k, n)+P_{e, S D}(k, n) P_{c, S R}(k, n)\right]},
$$

whereas the overall stability region, obtained by summing (14) over all $i \in\{A, B\}$, is given by

$$
\begin{aligned}
\Lambda_{R}^{C C}=\{ & \left(\lambda_{A}, \lambda_{B}\right):\left(\lambda_{A}+\lambda_{B}\right)< \\
& \left.\frac{\left[P_{c, S D}(k, n)+P_{e, S D}(k, n) P_{c, S R}(k, n)\right] P_{c, R D}(k, n)}{\left[P_{c, R D}(k, n)+P_{e, S D}(k, n) P_{c, S R}(k, n)\right]}\right\} .
\end{aligned}
$$

The overall stability region is given by the union of (11) and (15). However, by comparing (11) and (15) we observe that $\Lambda_{R}^{C C} \subseteq \Lambda_{S}^{C C}$, thus, the overall stability region $\Lambda^{C C}=\Lambda_{R}^{C C}$.

ii) The second statement is obtained by multiplying both sides of (15) with $k / n$ and maximizing with respect to $k$.

Although the optimization problem defined in (8) is not necessarily concave, it can be evaluated via exhaustive search. This does not introduce any additional computational complexity, due to the integer nature of the optimization problem.

The Non Cooperative (NC) protocol (absence of the relay terminal), is a special case of the cooperative protocol, with $P_{c, S R}(k, n)=0$. The overall stability condition is identical to the overall stability condition of source terminals, given by (11), where $P_{c, S R}(k, n)=0$, which yields the following corollary.

Corollary 1. For a fixed blocklength $n$, the overall stability condition of the non cooperative system is given by

$$
\left(\lambda_{A}+\lambda_{B}\right) \frac{k}{n}<\max _{k} u^{N C}(k, n) \triangleq u^{N C, *}(k, n),
$$

where $u^{N C}(k, n) \triangleq \frac{k}{n} P_{c, S D}(k, n)$. 


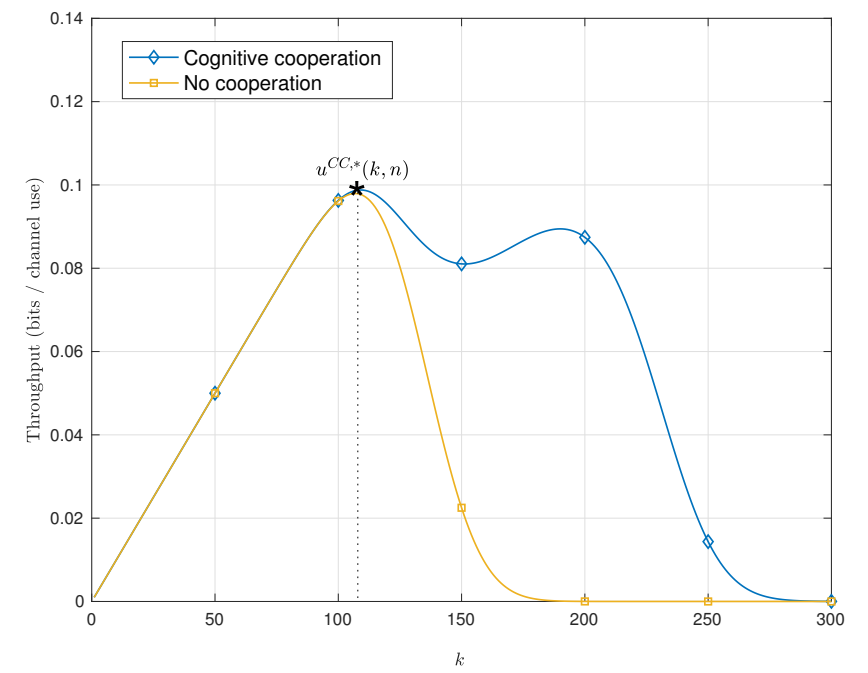

(a)

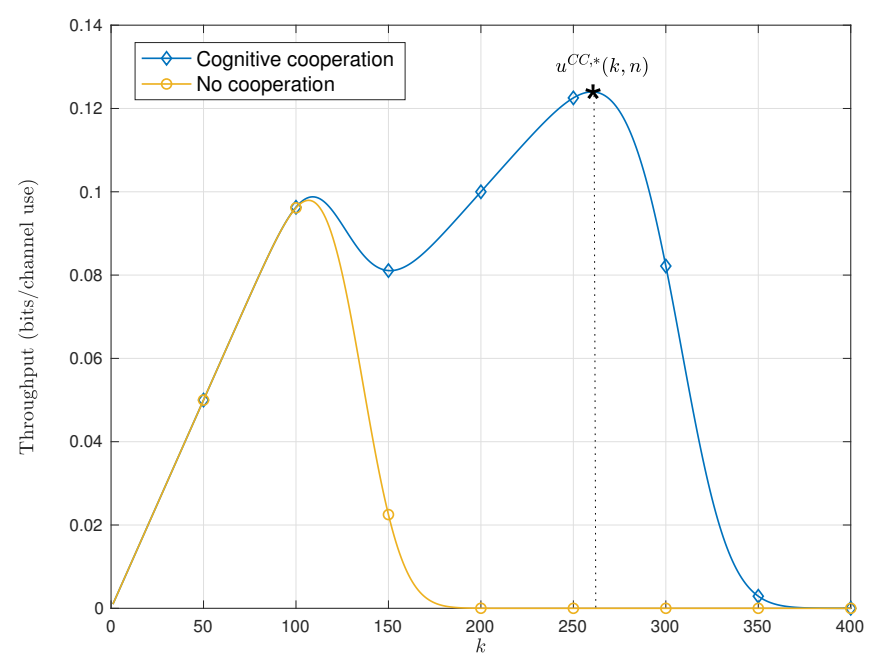

(b)

Fig. 2: Throughput for the non cooperative scheme and the cognitive cooperation scheme, for fixed blocklength $n=1000$. The channels from the source to the destination, from the source to the relay, and from the relay to the destination, are AWGN with SNR for (a) $0.2,0.35$ and 1, respectively, and for (b) $0.2,0.5$ and 1, respectively.

Remark 1. The performance, in terms of maximum throughput, of the CC protocol described in Definition 1, is not always better than the performance of the NC protocol. For example assume $P_{c, S D}(k, n)>0, P_{c, S R}(k, n)>0$ and $P_{c, R D}(k, n)=$ 0 . Then, from (6) and (16) we have $u^{C C, *}(k, n)=0$ and $u^{N C, *}(k, n)>0$. However, with some minor modifications of the CC protocol, we can guarantee that its performance is always better or at least equal to the performance of the NC protocol.

The optimal throughput of the non cooperative scheme, $u^{N C, *}(k, n)$, and the optimal throughput of the cognitive cooperation scheme, $u^{C C, *}(k, n)$, for two different channel triplets, is given in Fig. 2. For the selected $S N R$ triplet that is depicted in Fig. 2(a), the increase of the throughput due to cooperation is negligible, whereas for the $S N R$ triplet depicted in Fig. 2(b), cooperation increases throughput approximately by $25 \%$. However, taking into consideration the commitment of additional resources (relay, buffer memories and channels), the gain in the performance that cognitive cooperation exhibits over the non cooperative scheme, cannot be characterized as satisfactory. Comments for the insufficient performance of the cognitive cooperation protocol are given in the following remark.

Remark 2. The expression of the throughput for the overall system, $u^{C C}(k, n)$, involves the statistical characteristics (SNR) of all available channels. Optimizing throughput with respect to $k$, results in an optimal data packet size $k^{*}$, that is employed both from the source terminal and the relay. Thus, $k^{*}$ emerges as a compromise between the different statistics of those channels. This is directly reflected on the performance of the overall network, since, different channels pack the same amount of data into the codeword of fixed length $n$. An obvious solution to this problem is to allow the source terminal and the relay to pack different amount of data into the codeword (e.g. source packs $k_{S}$ bits into the codeword while the relay packs $k_{R}$ bits into the codeword), however, this is highly impractical, since, it introduces significant amount of complexity to the destination.

\section{COGNITIVE COOPERATION VIA BATCH AND FORWARD}

Motivated by the insufficient performance of the cognitive cooperation protocol, we propose a novel strategy that addresses the concerns encapsulated in Remark 2, and boosts the performance of the cognitive cooperation protocols in the finite blocklength regime. We evaluate the performance of the proposed strategy for the particular cognitive cooperation protocol given in Definition 1.

The proposed Batch-And-Forward (BAF) strategy, depicted in Fig. 3, keeps the data packet size the same for all individual nodes of the network, however, each node is allowed to batch more than one data packets into the codeword of length $n$. The number of data packets that are batched into the fixed length codeword, is denoted by $L$. Thus, this approach exploits the individual statistical characteristics of the different channels of the network, without imposing additional complexity on the overall scheme.

Next, we embed the BAF strategy at the relay of the cognitive cooperation protocol, and evaluate the performance of the overall network. This is implemented by replacing step vi) of Definition 1 with the following step. 


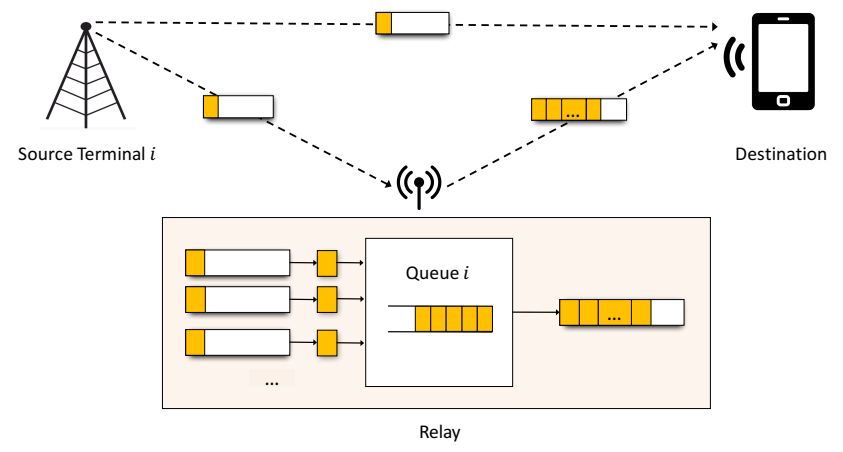

Data packet of

length $k$ Codeword of length $n$ with a data packe of length $k$

Fig. 3: The Batch-And-Forward strategy for a source terminal $i$-relay pair.

vi) The relay batches $L$ data packets from the queue $Q_{i R}, i \in$ $\{A, B\}$, and encodes them into a codeword of length $n$. When the source terminal $i \in\{A, B\}$ gains access to the channel, and it has no data packets in its queue (queue is idle), the relay transmits the codeword consisting of the $L$ data packets to the destination. If there are less than $L$ data packets in the respective queue at the relay, the relay does not transmit any information.

All the other procedures of Definition 1 do not change.

Theorem 2. Suppose that the relay employ the BAF strategy and let $u^{B A F}(L k, n)$ denote the overall throughput of cooperative scheme. Then, for a fixed blocklength $n$ :

i) the stability region of the BAF cooperative scheme satisfies

$$
\left(\lambda_{A}+\lambda_{B}\right) \frac{k}{n}<u^{B A F}(L k, n),
$$

where $k=1,2, \ldots, L=1,2, \ldots$, and

$$
\begin{aligned}
& u^{B A F}(L k, n)=\min \left\{u_{S}^{C C}(k, n), u_{R}^{B A F}(L k, n)\right\}, \\
& u_{S}^{C C}(k, n)=\frac{k}{n}\left[P_{c, S D}(k, n)+P_{e, S D}(k, n) P_{c, S R}(k, n)\right], \\
& u_{R}^{B A F}(L k, n)= \\
& \frac{L k}{n} \frac{\left[P_{c, S D}(k, n)+P_{e, S D}(k, n) P_{c, S R}(k, n)\right] P_{c, R D}(L k, n)}{\left[P_{c, R D}(L k, n)+P_{e, S D}(k, n) P_{c, S R}(k, n)\right]} .
\end{aligned}
$$

ii) the code rate of the overall scheme, $X^{B A F}(k, n)$, is bounded above by the maximum throughput $u^{B A F, *}(k, n)$, that is

$$
X^{B A F}(k, n)<u^{B A F, *}(k, n),
$$

where

$$
u^{B A F, *}(L k, n)=\max _{L, k} \min \left\{u_{S}^{C C}(k, n), u_{R}^{B A F}(L k, n)\right\} .
$$

Proof. i) The stability region for the source terminals is identical to the cognitive cooperation case, thus is given by (19). Similarly, the arrival rate at the relay terminal, is also identical with the arrival rate of the cognitive cooperation scheme, and is given by (12). Since $L$ packets are batched together, the total size of the data packets that are encoded into a codeword of length $n$, is $L k$. The departures from the queue $Q_{i R}, i \in\{A, B\}$ at the relay, are also Bernoulli distributed, with departure probability, at a given time slot, $q_{i R}=\omega_{i}\left(\pi_{i, 0}\right) P_{c, R D}(L k, n), i \in\{A, B\}$. The average departure rate is therefore given by

$$
\mu_{i R}=L q_{i R}=L \omega_{i}\left(\pi_{i, 0}\right) P_{c, R D}(L k, n), i \in\{A, B\} .
$$

The appropriate model at each queue of the relay is the Geo $/ \mathrm{Geo}^{L} / 1$ model [15]. In this model both the arrivals and the departures to and from the relay are Bernoulli distributed, and packets depart in batches of size $L$. The stability condition for $G e o / G^{L} o^{L} / 1$ queue [15], is given by

$$
\frac{\lambda_{i R}}{\mu_{i R}}<L, \quad \forall i \in\{A, B\} .
$$

By substituting (12) and (23) in (24), and by summing over $i \in\{A, B\}$, we obtain (20). Then, the stability of the overall network is given by the union of (19) and (20), which yields (18).

ii) The maximum throughput of the system is obtained by maximizing (18) with respect to $k$ and $L$.

The performance of the cognitive cooperation protocol with BAF strategy at the relay, is illustrated in Fig. 4. For the selected $S N R$ triplet depicted in Fig. 4(a), the optimal data packet size is 182 bits, whereas for the selected SNR triplet depicted in Fig. 4(b), the optimal data packet size is 227 bits. As it is depicted, the optimal batching size, for both cases, is $L=2$. It is also obvious that for both cases, the BAF strategy can significantly enhance the performance of the overall system (approximately by 75\%, in both scenarios), compared to the cognitive cooperation protocol without BAF. For both of the scenarios above, the $S N R$ of the channel between the relay and the destination, is higher than the $S N R$ of the channel between the source terminal and the destination, thus, is beneficiary for the overall performance of the network to apply the BAF strategy at the relay. For other scenarios in which the the $S N R$ of the channel between the source terminal and the destination, is higher than the $S N R$ of the channel between the relay and the destination, it would have been beneficial to have applied the BAF strategy at the source terminals. A multiple batching approach that can enhance the performance of more complex networks, is feasible by choosing different batching sizes for each node.

Though the majority of the classical cooperative techniques can be employed for short packet communication, they cannot fully correspond to the special characteristics of short codes, since, they were not particularly designed to perform optimally in the finite blocklength regime. The proposed approach, however, can significantly enhance the performance of the 


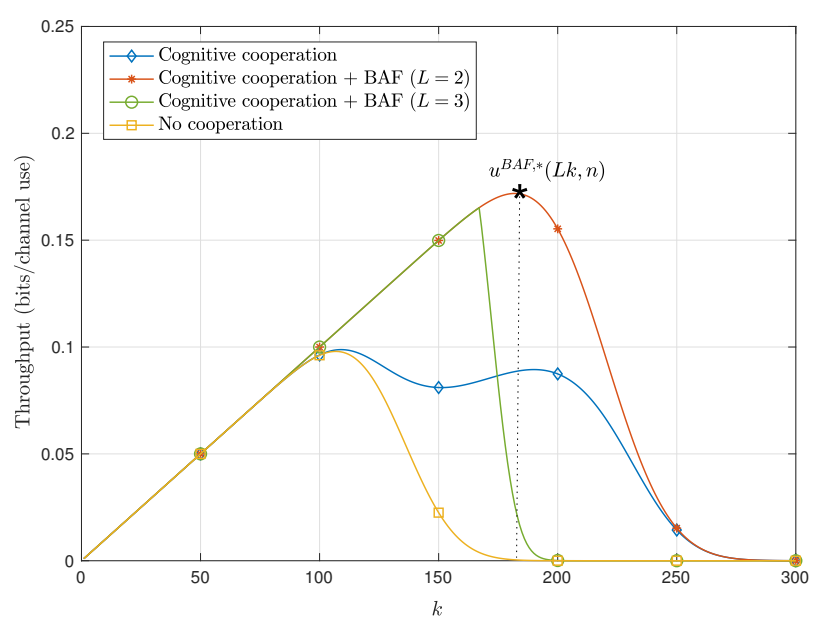

(a)

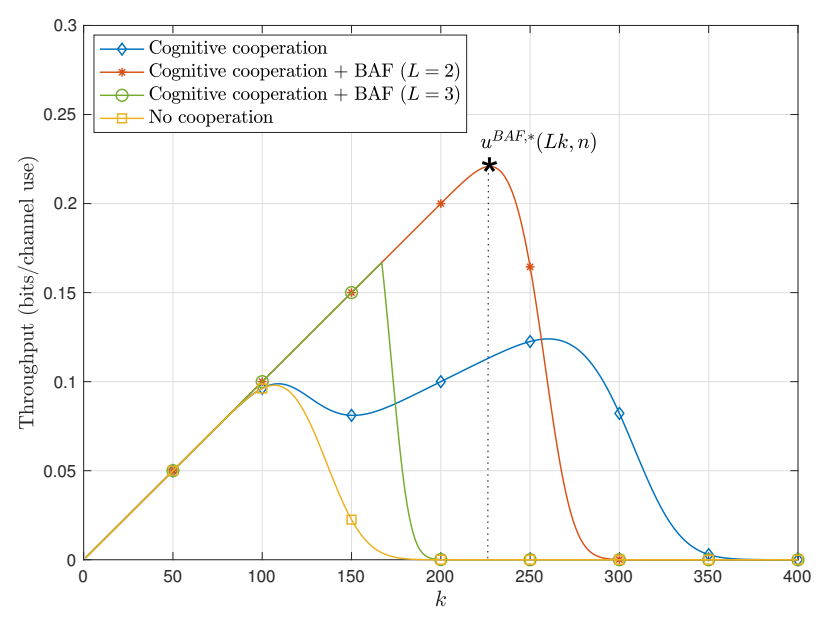

(b)

Fig. 4: Throughput of the cognitive cooperation protocol embedded with the BAF strategy, for fixed blocklength $n=1000$, and comparison with the no cooperation scheme and the cognitive cooperation protocol. The channels from the source to the destination, from the source to the relay, and from the relay to the destination, are AWGN with SNR for (a) 0.2, 0.35 and 1, respectively, and for (b) $0.2,0.5$ and 1 , respectively.

network, while at the same time it meets the finite blocklength requirements. Moreover, it can reduce the requirements in metadata, a challenging task in the actual implementation of short codes [2], since, it avoids the unnecessary repetition of metadata (e.g. address of the source terminal and the destination). Perhaps the most attractive feature, however, of the BAF strategy, is that it can be embedded into existing cooperative protocols, without imposing any additional complexity to the system.

\section{CONCLUSion}

In this work, we employed tools and results from information theory, stochastic processes and queueing theory, in order to provide a comprehensive framework regarding the analysis of the MARC network with TDMA scheduling, evaluated the stability conditions and the maximum throughput for a particular cognitive cooperation protocol, and proposed the BAF strategy that can significantly enhance the finite blocklength performance of cognitive protocols. In the current work we did not address issues regarding metadata, such as, impact of metadata on the performance and design of metadata for short codes. This is a challenging task for the performance analysis of URLLC, that will be investigated as a part of future work.

\section{REFERENCES}

[1] A. Ephremides and B. Hajek, "Information theory and communication networks: an unconsummated union," IEEE Transactions on Information Theory, vol. 44, no. 6, pp. 2416-2434, Oct. 1998.

[2] G. Durisi, T. Koch, and P. Popovski, "Toward massive, ultrareliable, and low-latency wireless communication with short packets," Proceedings of the IEEE, vol. 104, no. 9, pp. 1711-1726, Sept. 2016.

[3] Y. Polyanskiy, H. V. Poor, and S. Verdu, "Channel coding rate in the finite blocklength regime," IEEE Transactions on Information Theory, vol. 56, no. 5, pp. 2307-2359, May 2010.
[4] V. Y. F. Tan and M. Tomamichel, "The third-order term in the normal approximation for the awgn channel," IEEE Transactions on Information Theory, vol. 61, no. 5, pp. 2430-2438, May 2015.

[5] Y. Polyanskiy, H. V. Poor, and S. Verdu, "Feedback in the nonasymptotic regime," IEEE Transactions on Information Theory, vol. 57, no. 8, pp. 4903-4925, Aug. 2011.

[6] W. Yang, G. Durisi, T. Koch, and Y. Polyanskiy, "Quasi-static multipleantenna fading channels at finite blocklength," IEEE Transactions on Information Theory, vol. 60, no. 7, pp. 4232-4265, Jul. 2014.

[7] W. Yang, R. F. Schaefer, and H. V. Poor, "Finite-blocklength bounds for wiretap channels," in 2016 IEEE International Symposium on Information Theory (ISIT), July 2016, pp. 3087-3091.

[8] J. V. Wonterghem, A. Alloum, J. J. Boutros, and M. Moeneclaey, "On short-length error-correcting codes for 5g-nr," Ad Hoc Networks, vol. 79, pp. $53-62,2018$.

[9] R. Devassy, G. Durisi, P. Popovski, and E. G. Ström, "Finite-blocklength analysis of the arq-protocol throughput over the gaussian collision channel," in 2014 6th International Symposium on Communications, Control and Signal Processing (ISCCSP), May 2014, pp. 173-177.

[10] O. Simeone, Y. Bar-Ness, and U. Spagnolini, "Stable throughput of cognitive radios with and without relaying capability," IEEE Transactions on Communications, vol. 55, no. 12, pp. 2351-2360, Dec. 2007.

[11] A. K. Sadek, K. J. R. Liu, and A. Ephremides, "Cognitive multiple access via cooperation: Protocol design and performance analysis," IEEE Transactions on Information Theory, vol. 53, no. 10, pp. 36773696, Oct. 2007.

[12] I. Krikidis, J. N. Laneman, J. S. Thompson, and S. Mclaughlin, "Protocol design and throughput analysis for multi-user cognitive cooperative systems," IEEE Transactions on Wireless Communications, vol. 8, no. 9, pp. 4740-4751, Sep. 2009.

[13] R. A. Costa, M. Langberg, and J. Barros, "One-shot capacity of discrete channels," in 2010 IEEE International Symposium on Information Theory, Jun. 2010, pp. 211-215.

[14] V. Strassen, "Asymptotische abschätzungen in Shannons informationstheorie," 1962, pp. 689-723. [Online]. Available: http://www.math.cornell.edu/ $\sim$ pmlut/strassen.pdf

[15] A. S. Alfa, Queueing Theory for Telecommunications: Discrete Time Modelling of a Single Node System. Springer, 2010. 